ISSN: 2600-5859

\title{
Factores de riesgo mecánico en la extracción de aceite de palma: caso de la empresa La Joya
}

\author{
Mechanical risk factors in palma oil extraction: case of the La Joya company \\ Luis Javier Tapia Vasco. ${ }^{1}$, María Angélica Barreno Villacis. ${ }^{2}$, Edwin Ramiro Cevallos \\ Carvajal. ${ }^{3}$, Gabriela Beatriz Arias Palma. ${ }^{4} \&$ Manolo Alexander Córdova Suárez. ${ }^{5}$ \\ Recibidlo:05-01-2020 / Revisado:22-01-2020 /Aceptado: 03-02-2020/ Publicadlo: 05-03-2020
}

\begin{abstract}
DOI: https://doi.org/10.33262/concienciadigital.v3i1.2.1169

This research work determines the level of mechanical risks in the execution of palm oil extraction activities by workers in the company La Joya. The level of exposure, the probability of occurrence of the event and the consequences thereof were quantified using the William Fine method. To deepen the study, a statistical analysis of the accidents reported in 2017 was carried out using the Technical Note of Prevention (NTP) 236.

The investigation begins with the application of the matrix of identification of occupational hazards observing greater impact due to blows with mobile or immobile objects, falls at the same or different level, entrapment between objects, thermal contact, falling of detached objects, cuts and lacerations in the operators of palm oil extraction in the production area. Finally, a statistical analysis was applied with the application of the PJ Shipp tables, determining the months with the
\end{abstract}

1 Department of Food Science and Engineering, Technical University of Ambato, Ambato-Ecuador, lj.tapia@uta.edu.ec

${ }^{2}$ Faculty of Information Technology, Telecommunications and Industrial, Technical University of Ambato, AmbatoEcuador, angelicabarreno@hotmail.es

${ }^{3}$ Faculty of Agricultural Sciences and Natural Resources, Technical University of Cotopaxi, Latacunga-Ecuador, edwin.cevallos@utc.edu.ec

${ }^{4}$ Faculty of Agricultural Sciences and Natural Resources, Technical University of Cotopaxi, Latacunga-Ecuador, edwin.cevallos@utc.edu.ec

5 Department of Food Science and Engineering, Technical University of Ambato, Ambato-Ecuador, ma.cordova@uta.edu.ec 
highest incidence of accidents with the graphic comparison per month of the curve of the accumulated frequency indices and the upper and lower limits determined by the records of the 2017. The results determine a degree of danger (GP) of 120 in the position of: Red oil production boiler and in the digester or hopper, with a GP of 48 the sterilization and maintenance post.

Keywords: Mechanical risks, William Fine, frequency index, accident rate.

\section{Resumen}

El presente trabajo de investigación determina el nivel de riesgos mecánicos en la ejecución de actividades de extracción de aceite de palma por parte de los trabajadores en la empresa La Joya. Se cuantificó cuali cuantitativamente el nivel de exposición, la probabilidad de ocurrencia del evento y las consecuencias de los mismos utilizando el método de William Fine. Para profundizar el estudio se realizó un análisis estadístico de los accidentes reportados en el año 2017 utilizando la Nota Técnica de Prevención (NTP) 236.

La investigación empieza con la aplicación de la matriz de identificación de riesgos laborales observando mayor afectación por golpes con objetos móviles o inmóviles, caídas al mismo o a diferente nivel, atrapamiento entre objetos, contacto térmico, caída de objetos desprendidos, cortes y laceraciones en los operadores de extracción del aceite de palma en el área de producción. Finalmente se aplicó un análisis estadístico con la aplicación de las tablas de P. J. Shipp determinando los meses de mayor incidencia de los accidentes con la comparación gráfica por mes de la curva de los índices de frecuencia acumulada y los límites superior e inferior que determina los registros del 2017. Los resultados determinan un grado de peligrosidad (GP) de 120 en el puesto de: Caldera de producción de aceite rojo y en el digestor o tolva, con un GP de 48 el puesto de esterilización y mantenimiento (Barreno Villacís, 2019).

Palabras claves: Riesgos mecánicos, William Fine, índice de frecuencia, accidentabilidad.

\section{Introducción}

El estudio de los factores de riesgo mecánico y su impacto en los accidentes presentados durante el proceso de producción de aceite de palma africano se considera de gran importancia, debido a un incremento de fábricas e industrias de procesamiento de este tipo de alimento (Yánez Herdoiza, 2013). Esto conlleva a establecer las condiciones de riesgos que preceden las medidas correctivas para controlar las tasas de accidentes y el ausentismo laboral que influyen directamente en los costos de producción y en molestias por los paros inesperados (Apolo Romero, 2012). 
En la actualidad, la exigencia profesional en cuanto a higiene ocupacional recomienda significar los riesgos establecidos para mejorar la calidad de las condiciones del trabajo. La investigación de los elementos que determinan los escenarios laborales crean la necesidad de reformar el sistema de actuación del departamento de prevención de riesgos empresarial (Vásquez, Matheus, \& Chirinos, 2015), lo que obliga a utilizar métodos específicos para mejorará la valoración y no incurrir en gastos innecesarios por valores con mucha incertidumbre (Cardona Arboleda, 2008) especialmente en actividades que han sido tratadas como un conocimiento empírico que ha pasado de generación a generación y no se ha modificado para evitar la exposición a factores de riesgo mecánicos agresivos (Hurtado Calle \& Solís Guamán, 2015) en este tipo de actividad peligrosa como lo es el de extracción de aceite de palma (Rengifo \& Stalin, 2015).

Los métodos de evaluación de riesgos mecánicos son extensos (Romero, 2004) y la mayoría considera variables cuali cuantitativas. Entre los más conocidos se encuentran el método simplificado de evaluación de accidentes que se detalla en la NTP 330 y el método de William Fine. Aunque existen una clara diferencia entre los dos métodos en cuanto a los resultados no se ha establecido una diferencia significativa de su uso ya que la aplicación de los dos métodos dependen de apreciaciones subjetivas (Moya Palacios, 2016) sin embargo los métodos que utilizan matemática booleana como el árbol de fallos son más objetivos y dan un mejor resultado pero su aplicación depende de conocimiento de matemáticas más extenso limitando su aplicación mas no su validez (HAUPTMANS, 1979).

Los programas de atenuación de riesgos mecánicos están ligados a los resultados finales de los niveles de peligrosidad de cada puesto de trabajo con exposición. Una vez identificados los grados de afectación la intervención será más efectiva y los recursos serán mejor utilizados (Rodríguez Daza, 2015).

Las medidas correctivas se plasman en procedimientos de trabajo seguro que tiendan a controlar los factores preponderantes en el medio, la fuente y en el receptor según menciona la legislación Ecuatoriana (Laborales, 2004). La correcta aplicación de medidas de seguridad y control de actos y condiciones subestándar inciden directamente en gastos sombra y en costos directos que las empresas incurren por responsabilidades patronales (Gómez García, Algora Buenafé, Suasnavas Bermúdez, \& Vilaret Serpa, 2016). Este trabajo contempló la determinación de los factores 
ISSN: 2600-5859

importantes de riesgo mecánico y su acaecimiento en la frecuencia de accidentes laborales del área de producción de aceite de palma africana en la empresa La Joya en Ecuador.

\section{Metodología}

Materiales y Equipos: aunque para la determinación del índice riesgo mecánico se requiera de un estudio de trabajo de campo con el uso de medios audios visuales de grabación no se considera como un lineamiento imprescindible ya que su aplicación solo requiere de observación por parte del investigador. Sin embargo, se utilizó una cámara fotográfica SONY y seguir los lineamientos de la elección de las mejores herramientas para la prevención de riesgos (Rosauro, 2012).

\section{Método de análisis estadístico de accidentes laborales}

\section{NTP 236. Método de las líneas límite}

Este método es parte de la investigación de las causas de accidentes de trabajo, antes de recomendar medidas preventivas en los centros de trabajo (DE, 1998). Se basa en el estudio de la marcha del índice de frecuencia acumulado y determina si los cambios observados en la curva de frecuencia acumulada son resultado de las variaciones y la aleatoriedad de los datos o si son debido a la ocurrencia de algún nuevo elemento que cambia las condiciones de seguridad del sitio (González Tobar \& Castro Del Pozo, 2018).

El método de las líneas límites considera cálculos estadísticos para determinar las curvas de frecuencia acumulada y comprara con las líneas de tendencia superior e inferior que se escogió al inicio del cálculo según las tablas recomendadas junto a datos provenientes de otras fuentes. (Mazorra Olmedo, 2017). Los valores hacen referencia a las tablas de P. J. Shipp y permiten establecer, en función del número de horas trabajadas en millares hasta un límite de 1.200.000 horas y para un margen de confianza del 90\%. Nos muestra valores límite superiores e inferior considerando el índice de frecuencia esperado Ie hasta un límite de 175, ya sea éste el mismo del año anterior, o bien una determinada reducción del mismo fundada en una política de objetivos de prevención de riesgos laborales.(González, 2005)

\section{Método de evaluación de riesgos mecánicos}

Los riesgos mecánicos se estudian considerando los actos inseguros que ocasionarán el accidente laboral y las condiciones de las máquinas, equipos y entorno disponibles para su ejecución. Entre los métodos más utilizados y fáciles de aplicar están el de William Fine que originalmente se desarrolló para control de riesgos cuyas reparaciones son de alto costo. Es un método 
probabilístico que determina un grado de peligrosidad (GP) considerando una escala de valores para la consecuencia (C), la exposición (P) y la probabilidad (P)(Morales Perrazo, 2013). La multiplicación de estas variables determina la magnitud del riesgo final. Ver ecuación (1).

$\mathrm{GP}=\mathrm{CxExP}$ Ecu. (1)

Donde:

$\mathrm{GP}=$ Grado de peligrosidad

$\mathrm{C}=$ Nivel de consecuencia

$\mathrm{E}=$ Nivel de exposición

Los valores de referencia se mencionan en la tabla 1, 2 y 3 en la descripción como parte de los métodos matemáticos para control de peligros. (Fine, 1971)

Tabla 1

Valor de consecuencia

\begin{tabular}{lc}
\hline \multicolumn{1}{c}{ Consecuencia } & Valor \\
\hline 1. Desastre: Numerosas fatalidades, daños grandes (superior a 1000 000 & 100 \\
USD), gran quebranto de la actividad. & 50 \\
2. Múltiples fatalidades: (Daños entre 500 000 a 1000 000 USD) & 25 \\
3. Fatalidad: (Daños entre 100000 a 5000 USD) & 15 \\
4. Lesiones sumamente graves (Invalidez Permanente) (Daños de 1000 a & 5 \\
100 000 USD) & 1 \\
5. Lesiones con baja: (Daños hasta 1000 USD) & \\
6. Heridas menores, contusiones, golpes, pequeños daños &
\end{tabular}

Nota: Adaptado de los métodos matemáticos para evaluar peligros.

Se definen seis niveles de consecuencia con su respectiva ponderación. (Fine, 1971)

Tabla 2

Valor de exposición

\begin{tabular}{lc}
\hline \multicolumn{1}{c}{ EXPOSICIÓN } & VALOR \\
\hline 1. Consecutivamente, muchas veces al día & 10 \\
2. Frecuentemente, una vez por día & 6 \\
3. En ocasiones de una vez por semana a una al mes & 3 \\
4. Discontinuamente de una vez al mes a una vez al año & 3 \\
5. Raramente & 1 \\
6. Remotamente posible & 0.5 \\
\hline
\end{tabular}

Nota: Adaptado de los métodos matemáticos para evaluar peligros.

Se definen seis niveles de exposición con su respectiva ponderación. (Fine, 1971) 
ISSN: 2600-5859

Tabla 3

Valor de probabilidad

\begin{tabular}{ll}
\hline \multicolumn{1}{c}{ PROBABILIDAD } & VALOR \\
\hline 1. Lo más probable y esperado si se presenta el riesgo & 10 \\
2. Completamente posible (probabilidad del 50\%) & 6 \\
3. Seria consecuencia o consecuencia rara & 3 \\
4. Consecuencia remotamente posible, se sabe ha ocurrido & 1 \\
5. Extremadamente remota, pero concebible & 0.5 \\
6. Prácticamente imposible, 1 en un millón & 0.1
\end{tabular}

Nota: Adaptado de los métodos matemáticos para evaluar peligros.

Se definen seis niveles de probabilidad con su respectiva ponderación. (Fine, 1971)

Las combinaciones de los eventos con cada elección determinan un rango de actuación considerado en tres niveles de prioridad. Ver tabla 4.:

Tabla 4

Valor del grado de peligrosidad

\begin{tabular}{ll}
\hline Grado de peligrosidad & Actuación \\
\hline De 270 a 1500 & $\begin{array}{l}\text { Necesita corrección inmediata. La actividad debe ser detenida } \\
\text { hasta que el riesgo se haya bajado. }\end{array}$ \\
De 90 a 269 & $\begin{array}{l}\text { Urgente. El puesto requiere atención lo antes posible. } \\
\text { De } 18 \text { a } 89\end{array}$ \\
& El riesgo debe ser atenuado sin demora, pero la situación no es \\
& una emergencia.
\end{tabular}

Nota: Adaptado de los métodos matemáticos para evaluar peligros.

Se definen seis niveles de probabilidad con su respectiva ponderación. (Fine, 1971)

\section{Resultados}

\section{Resultado de la evaluación de riesgo mecánico}

Luego de realizar el estudio de trabajo y el análisis de campo en el área de producción de aceite de palma de la empresa "La Joya" se obtuvieron los siguientes resultados. 
ISSN: 2600-5859

Tabla 5

Evaluación de riesgo mecánico

\begin{tabular}{|c|c|c|c|c|c|}
\hline Área & Puesto de trabajo & GP & $\begin{array}{c}\text { Nivel de } \\
\text { peligrosidad }\end{array}$ & $\begin{array}{l}\text { Riesgo } \\
\text { potencial }\end{array}$ & Accidente \\
\hline Digestor & Tolvero & 1150 & Alto & $\begin{array}{l}\text { Caída a } \\
\text { distinto nivel }\end{array}$ & Fractura de pie \\
\hline Esterilización & $\begin{array}{l}\text { Operario de } \\
\text { esterilización }\end{array}$ & 436 & Medio & $\begin{array}{l}\text { Altas } \\
\text { temperatura }\end{array}$ & $\begin{array}{l}\text { Quemadura de } \\
\text { 2do grado }\end{array}$ \\
\hline Caldera 1 & $\begin{array}{l}\text { Calderista de aceite } \\
\text { rojo }\end{array}$ & 1900 & Alto & Atrapamiento & $\begin{array}{l}\text { Pérdida de } \\
\text { miembros } \\
\text { superiores }\end{array}$ \\
\hline Caldera 2 & $\begin{array}{l}\text { Calderista } \\
\text { palmistería }\end{array}$ & 1900 & Alto & Aplastamiento & $\begin{array}{l}\text { Pérdida de } \\
\text { miembros } \\
\text { superiores }\end{array}$ \\
\hline Mantenimiento & Mecánico & 434 & Medio & Atrapamiento & Corte de brazo \\
\hline
\end{tabular}

Luego del análisis de los factores de riesgo mecánicos al puesto de trabajo en los procesos de extracción de aceite rojo y extracción de aceite de palmiste, se identificaron las condiciones inseguras detectándose en el área de esterilización exposición a altas temperaturas $\left(147^{\circ} \mathrm{C}\right)$ por la falta de un mantenimiento ya que no se remplazaron los empaques de retención de temperatura de la góndola de cocción. En el área de caldera 1 del proceso de aceite rojo se encontraron como fuentes de riesgo maquinarias desprotegidas y motores sin cubre bandas exponiendo continuamente a que el trabajador introduzca sus miembros superiores con presencia de motores encendidos y sin protección al realizar la limpieza correspondiente. El área de caldera 2 del proceso de palmisteria se encontró como causa raíz la falta de holgura en el puesto para para realizar sus actividades laborales, pudiendo originar en el operario aplastamiento de sus extremidades superiores al levantar el ducto del silo de almendra.

\section{Resultado del análisis de accidentabilidad}

$\mathrm{Al}$ aplicar el método de la NTP 236 se determinó los siguientes resultados descritos en la tabla 6. 
ISSN: 2600-5859

Tabla 6

Accidentabilidad 2017

\begin{tabular}{|c|c|c|c|c|c|}
\hline Área & $\begin{array}{l}\text { Puesto de } \\
\text { trabajo }\end{array}$ & Accidentes & $\begin{array}{c}\text { Días } \\
\text { perdidos }\end{array}$ & $\begin{array}{c}\text { Índice de } \\
\text { frecuencia }\end{array}$ & $\begin{array}{l}\text { Índice de } \\
\text { gravedad }\end{array}$ \\
\hline Enero & 1024 & & & & \\
\hline Febrero & 9600 & 1 & 60 & 97,7 & 100,00 \\
\hline Marzo & 9760 & & & & \\
\hline Abril & 10240 & & & & \\
\hline Mayo & 10080 & & & & \\
\hline Junio & 9600 & & & & \\
\hline Julio & 9600 & & & & \\
\hline Agosto & 10206 & 1 & 45 & 97,8 & 132,27 \\
\hline Septiembre & 10206 & & & & \\
\hline Octubre & 9600 & 1 & 45 & 92,3 & 140,60 \\
\hline Noviembre & 10206 & 1 & 30 & 98 & 84,00 \\
\hline Diciembre & 118938 & 5 & 195 & 41,9 & 45,05 \\
\hline
\end{tabular}

Nota: Adaptado de resultados de: "Factor de riesgo mecánico y su influencia en los accidentes de trabajo de los operadores de extractoras de aceite de palma africana" En las incapacidades permanentes parciales se registraron pérdidas de dedos (primera y segunda falange) pulgar, anular en los dos casos. Los demás accidentes se categorizaron con bajas laborales por períodos de reposos superiores a las 24 horas (Barreno Villacís, 2019)

\section{Conclusiones}

Para el año 2017 a través del método NTP 236, se calcula el índice de frecuencia (IF) y en el índice de gravedad (IG) para control estadístico de accidentabilidad en la empresa, dando como resultado los siguientes datos, el índice de frecuencia es de 41.9 con los datos obtenidos se puede tener un control estadístico para detectar, a través de la evolución del índice de frecuencia. Si los cambios experimentados son debidos a una fluctuación aleatoria o a la entrada de un nuevo factor que ha modificado las condiciones de seguridad. El índice de gravedad obtenido en la empresa es de 42.33 tomando en cuenta las incapacidades permanentes tanto parciales como totales anexadas al dividendo el producto del valor obtenido de la tabla de baremo (NTP-1). 
ISSN: 2600-5859

\section{Referencias bibliográficas}

Apolo Romero, O. A. (2012). Elaboración de un Manual de Procedimientos de Seguridad Industrial para el Proceso de Extracción de Aceite de Palma y Almendra en La Empresa. Escuela Superior Politécnica de Chimborazo.

Barreno Villacís, A. M. (2019). Factores de riesgos mecánicos y su influencia en los accidentes de trabajo de los operadores de extractoras de aceite de palma africana. Universidad Técnica de Ambato. Facultad de Ingeniería en Sistemas ....

Cardona Arboleda, O. D. (2008). Medición de la gestión del riesgo en América Latina.

DE, A. O. D. T. C. (1998). Seguridad e higiene en el trabajo.

Fine, W. T. (1971). Mathematical evaluations for controlling hazards: NAVAL ORDNANCE LAB WHITE OAK MD.

Gómez García, A. R., Algora Buenafé, A. F., Suasnavas Bermúdez, P. R., \& Vilaret Serpa, A. (2016). Notificación de Accidentes de Trabajo y Posibles Enfermedades Profesionales en Ecuador, 2010-2015. Ciencia \& trabajo, 18(57), 166-172.

González, C. A. R. (2005). Análisis estadístico de los daños derivados del trabajo. Manual para la formación de nivel superior en prevención de riesgos laborales, 75.

González Tobar, E. A., \& Castro Del Pozo, H. A. (2018). Prevención de riesgos en establecimientos educativos en base al Decreto Ejecutivo 2393 del Distrito Zona 8 de la parroquia Ximena. Universidad Estatal de Bolívar. Facultad de Ciencias de la Salud y del Ser ....

HAUPTMANS, U. (1979). METODOS PARA LA EVALUACION DE ARBOLES DE FALLOS.

Hurtado Calle, C. L., \& Solís Guamán, F. F. (2015). Elaboración del plan de seguridad industrial con base en la identificación y evaluación de los riesgos laborales del taller de Ingeniería Mecánica Automotriz de la Universidad Politécnica Salesiana.

Laborales, M. (2004). Reglamento de Seguridad y Salud de los Trabajadores y Mejoramiento del Medio Ambiente de Trabajo. Decreto Ejecutivo (2393).

Mazorra Olmedo, F. X. (2017). Riesgo Mecánico y su incidencia en la salud de los trabajadores del Área de Talleres del Gobierno Autónomo Descentralizado Provincial de Pastaza. Universidad Técnica de Ambato. Facultad de Ingeniería en Sistemas .... 
ISSN: 2600-5859

Morales Perrazo, L. A. (2013). Riesgos mecánicos y su influencia en la seguridad laboral de la planta de producción en la empresa "PASTIFICIO AMBATO CA. Universidad Técnica de Ambato. Facultad de Ingeniería en Sistemas ....

Moya Palacios, K. A. (2016). Análisis comparativo entre la metodología NTP 330 Y WILLIAM FINE para la gestión de riesgos mecánicos en el área de producción de la Empresa INOX Industrial. Escuela Superior Politécnica de Chimborazo.

Rengifo, R., \& Stalin, L. (2015). Determinantes de riesgo y exposición a factor de riesgo mecánico en la producción agroindustrial de aceite de palma. POSGRADOS FACULTAD: MAESTRÍA EN SEGURIDAD Y PREVENCIÓN DE RIESGOS DEL TRABAJO.

Rodríguez Daza, G. C. (2015). Diseño de procedimientos e instructivos para implementar buenas prácticas de manufactura en el proceso de extracción de aceite en palma aceitera Elaeis guineensis Jacq. San Lorenzo, Esmeraldas. Quito: UCE.

Romero, J. C. R. (2004). Métodos de evaluación de riesgos laborales: Ediciones Díaz de Santos. Rosauro, F. L. (2012). Prevención de riesgos laborales: Editorial Vértice.

Vásquez, A. J. M., Matheus, M. M. R., \& Chirinos, Á. A. U. (2015). Gestión de riesgos en la empresa RC Agelvis, CA. Multiciencias, 15(4), 417-427.

Yánez Herdoiza, M. G. (2013). ESTUDIO DE FACTIBILIDAD PARA LA EXPORTACIÓN DE ACEITE DE PALMA A INDIA.

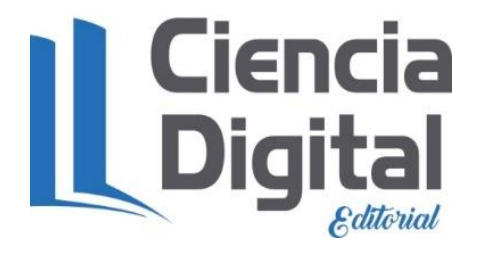


ISSN: 2600-5859

\section{PARA CITAR EL ARTÍCULO INDEXADO}

Tapia Vasco, L. J., Barreno Villacis, M. A., Cevallos Carvajal, E. R., Arias Palma, G. B., \& Córdova Suárez, M. A. (2020). Factores de riesgo mecánico en la extracción de aceite de palma: caso de la empresa La Joya. ConcienciaDigital, 3(1.2), 33-43. https://doi.org/10.33262/concienciadigital.v3i1.2.1169



El artículo que se publica es de exclusiva responsabilidad de los autores y no necesariamente reflejan el pensamiento de la Revista Conciencia Digital.

El artículo queda en propiedad de la revista y, por tanto, su publicación parcial y/o total en otro medio tiene que ser autorizado por el director de la Revista Conciencia Digital.
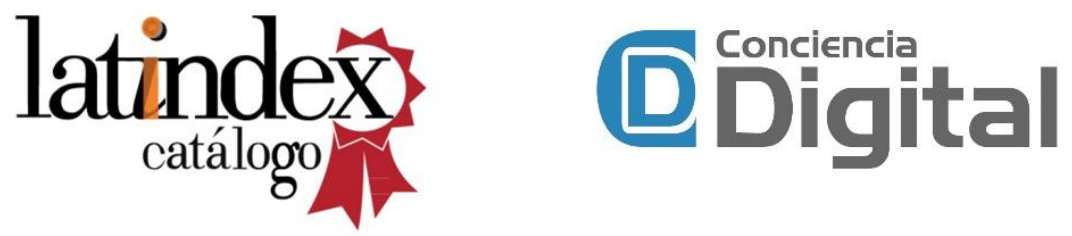